\title{
Laparoscopic Management of Chronic Pelvic Abscess which Turned out to be Textilloma or Gossypiboma
}

\author{
Bindra V*, Das PJ and Sarada P \\ Department of Obstetrics and Gynaecology and \\ Department of Radiology, Apollo Hospitals, Telangana, \\ India \\ *Corresponding author: Vimee Bindra, Department \\ of Obstetrics and Gynaecology and Department of \\ Radiology, Apollo Hospitals, Telangana, India
}

Received: November 30, 2018; Accepted: December 27, 2018; Published: December 31, 2018

\begin{abstract}
Textilloma or Gossypiboma refers to retained sponges or swabs, although rare but does happens in practice due to various causes and also we come across such cases rarely in literature due to underreporting of these cases. For any surgical procedure first aim of surgeon is achieving complete hemostasis either by electrosurgical sources or using cotton sponges and swabs. Here we are presenting a case of 67 year old woman who presented with pus coming down per vaginum for three months and was managed laparoscopically by removal of gossypiboma. The purpose of reporting this case is requirement of more vigilance and avoidance of such instances.
\end{abstract}

Keywords: Laparoscopy; Gossypiboma; Textilloma

\section{Introduction}

Textilloma or gossypiboma is defined as retained cotton sponges or swabs in the body after surgery. It is a rare but ubiquitous medical error $[1,2]$. There is a reluctance to publish such cases because of medico-legal implications and criticism from public and medical professionals [3]. The higher incidence of textillomas is found in emergency surgeries, when multiple surgical teams are involved, huge intra-operative bleeds, unexpected intra-operative findings, and incorrect counts. Even after introduction of check lists and other precautions, it happens. It can have variable presentation such as abscess, sepsis, tumour, and also can be asymptomatic for years together [4]. We describe a case of intra-abdominal textilloma mimicking an abscess and soft tissue tumour on CT scan. Retained foreign body should be kept in mind when there is a resistant abscess not responding to any treatment and spongiform lesion on CT scan may help in pre-operative diagnosis and assessment.

\section{Case Presentation}

This is a retrospective case report and patient informed consent was taken for publication and use of pictures. Being a retrospective case study, this does not need IRB approval.

A 67 year old woman, P4L4, was referred to our centre with a presentation of bleeding and pus oozing from vagina for last three months. She was being treated with a course of antibiotics for infection. She had undergone two surgeries at two different centres outside. One was laparoscopy for pelvic pain two years back and another surgery was Total Abdominal Hysterectomy with bilateral salpingo-oophorectomy for abnormal uterine bleeding, 4 months back in a centre outside. She was absolutely fine for one month after surgery and started experiencing some discharge from vagina after one month of surgery and was treated conservatively for the same. When she stopped responding to all treatment outside, she was referred to our centre for management.

On examination, per abdomen it was soft, on speculum examination, frank pus was coming out from the vault and on per vaginum examination, it was a boggy feeling. She underwent ultrasound which showed "Vague hypoechoic area measuring 7.5 $\mathrm{x} 5 \mathrm{~cm}$ with dirty shadowing is noted posterior to urinary bladder? Collection to air pockets? Foreign body with collection". For confirmation she underwent CT scan with contrast which showed an ovoid heterogeneous lesion of approximately $75 \times 42 \mathrm{~mm}$ is noted along the vaginal vault with air foci within and a thick enhancing rim. The walls of the lesion appear continuous with the vaginal walls (Figure 1). The lesion is abutting closely the urinary bladder and rectosigmoid region, with mild adjacent wall thickening of both these structures. There is mild surrounding fat stranding - possibly infective pathology.

On laparascopy sigmoid colon adherent to anterior abdominal wall, flimsy adhesions between loops of Bowel present, 7 x $5 \mathrm{~cm}$ loculated \& encapsulated abscess present at Vault (Figure 2), adhesiolysis done, abscess decapsulated and pus drained out \& sent for culture and sensitivity. In between pus there was found retained surgical gauze and diagnosis of textilloma was confirmed. The sponge was removed through the $10 \mathrm{~mm}$ port in an endobag (Figure 3 and 4 ).

Complete peritoneal saline wash given, hemostasis achieved. Intraabdominal drain kept in situ. Her postoperative period was uneventful. Pus was sent for culture sensitivity and it was found gram negative rods and she was given specific antibiotics and patient recovered well. After 5 days patient was discharged and advice to follow up.

\section{Discussion}

A chronic infective case like this can be a diagnostic dilemma for soft tissue tumours, infective pathologies. Textilloma should be kept in differential diagnosis for such kind of presentation for patients who have undergone abdominal surgeries. Due to non-specific picture early disgnois is difficult and increases patient morbidity [3]. Two major types of reaction can occur in response to surgical foreign body first is an abscess may form with or without secondary infection. The second reaction is an aseptic fibrinous response with may cause encapsulation and formation of foreign body granuloma [4]. 


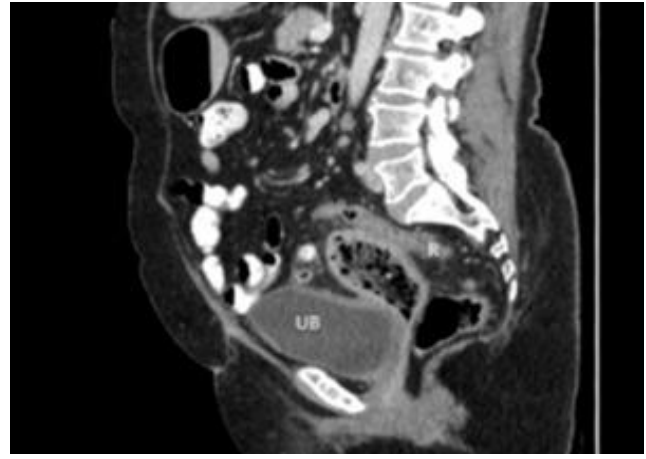

Figure 1: CT scan picture showing mass or abscess with air pockets.

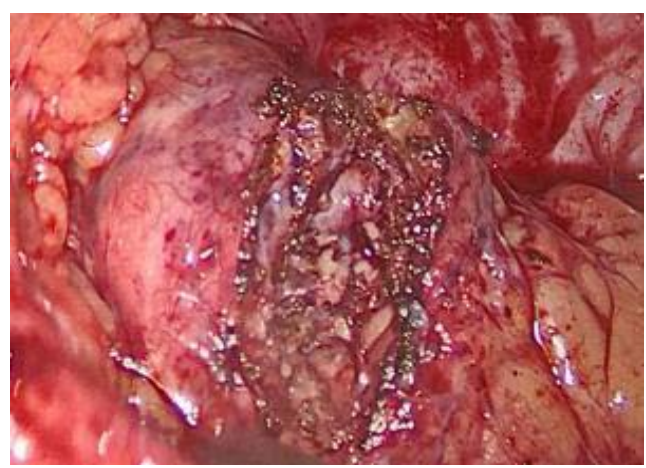

Figure 2: Encapsulated sponge forming a gossypiboma or textilloma.

The gossypiboma cases can lead to embarrassment, humiliation, loss of job, and law suit worldwide. Data presenting the actual incidence is difficult to estimate because of under-reporting of such cases [5]. It varies between 1 in 1000-1500 of intra-abdominal surgeries to 1 in 300-1000 of all surgeries [6]. The possibility of a retained foreign body should be kept in differential diagnosis who present with pain, infection, or palpable mass [7].

The possible risk factors for retained sponge are emergency surgeries, unexplained change in surgical procedure, and involvement of two or more surgical teams, dissorganisation (miscommunication), hurried sponge count, long operations, inexperienced staff, inadequate staff and obesity. NEJM (New England Journal of Medicine) published an article about the risk factors for retained foreign body. Of the 8 risk factors, authors identified only 3 factors (unplanned change in the operation, emergency surgery and BMI) to be statistically significant by multivariate logistic analysis. The counting of sponges and instruments was not a significant factor [2].

Any patient with chronic pain, infection with a history of prior surgery, gossypiboma should be kept in the differential diagnosis. This can be avoided by use of radio-opaque sponges, avoiding small gauze pieces in open surgeries, trained staff and the operating surgeon should check the count themselves with the help of scrub nurses.

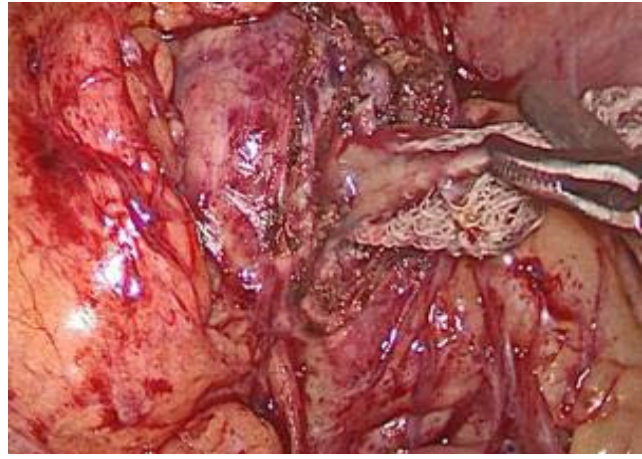

Figure 3: Sponge being removed with pus that was the reason for chronic vaginal discharge.

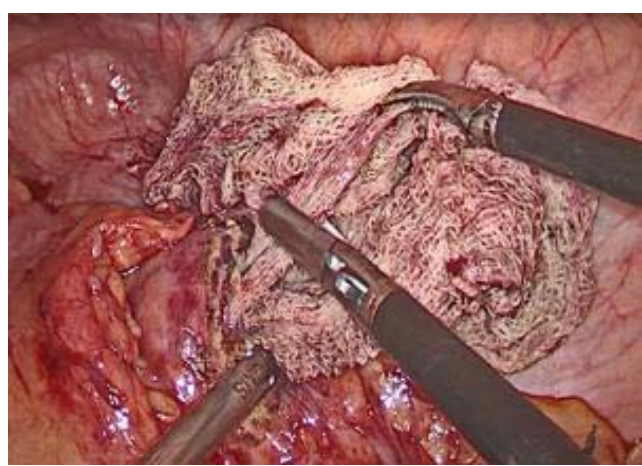

Figure 4: Sponge being removed laparoscopically.

\section{Acknowledgment}

We acknowledge the contribution of our hospital staff and nursing staff for all the help for the surgical procedure.

\section{References}

1. Montasser AM, Eman T, Edward PM. The Recurring Problem of Retained Swabs and Instruments. Best Pract Res Clin Obstet Gynaecol. 2013; 27 : 489-495.

2. Gawande AA, Studdert DM, Orav EJ, et al. Risk factors for Retained Instruments and Sponges after Surgery. N Engl J Med. 2003; 348: 229-235.

3. MacIntye LK, Jurkovich GJ, Gunn. ML, et al. Gossypiboma: Tales of Lost Sponges and Lessons Learned. Arch Surg. 2010; 145: 770-775.

4. Shyung LR, Chang WH, Lin SC, Shih SC, Kao CR, Chou SY. Report of gossypiboma from the standpoint in medicine and law. World J Gastroenterol. 2005; 11: 1248-1249.

5. Uluçay T, Dizdar MG, SunayYavuz M, Aşirdizer M. The importance of medico-legal evaluation in a case with intraabdominal gossypiboma. Forensic Sci Int. 2010; 198: e15-18.

6. Lincourt AE, Harrell A, Cristiano J, Schrist C, Kercher K, Heniford BT. Retained foreign bodies after surgery. J Surg Res. 2007; 138: 170-174.

7. Indu Lata. Gossypiboma, a rare cause of acute abdomen: A case report and review of literature. Int J Crit IIIn Sci. 2011; 1: 157-160.
Austin J Clin Case Rep - Volume 5 Issue 3 - 2018 ISSN : 2381-912X | www.austinpublishing group.com Bindra et al. () All rights are reserved
Citation: Bindra V, Das PJ and Sarada P. Laparoscopic Management of Chronic Pelvic Abscess which Turned out to be Textilloma or Gossypiboma. Austin J Clin Case Rep. 2018; 5(3): 1136. 